\title{
The Performance of Presented Subpixel Corner Detection Approach in Context of Camera Stereo Pair Displacement Determination
}

\author{
Lukáš Šroba*, Ján Grman and Rudolf Ravas \\ Institute of Electrical Engineering, Slovak University of Technology, Bratislava, Slovakia \\ *Corresponding author
}

\begin{abstract}
This paper deals with the comparison of the presented approach to the another subpixel corner detectors. The our proposed and considered detection method uses the regular cornerness map obtained from the usual corner detectors as initial step. The main idea is to use the cornerness values from a neighboring area around found corner point as a $3 D$ profile object and to find the coordinates of its centroid. As the subject of this comparison the determination procedure of the mutual displacement between two corresponding cameras using image stereo pair and prior information coming from camera calibration was used. This whole process of distance estimation consists of multiple steps which are also briefly explained on the next pages. The all results were statistically analyzed, listed in a table and turned into specific findings and conclusions.
\end{abstract}

Keywords-subpixel corner detection; cornerness map; image stereo pair; $3 D$ reconstruction

\section{INTRODUCTION}

The paper slightly follows our previous papers where the idea of describing approach to refinement corner coordinates into subpixel space was firstly introduced [1]. Also the comparisons using tasks involving homography and reprojection from 3D reconstruction were already done [2]. Here the performance of our approach is tested on a complex $3 \mathrm{D}$ reconstruction task, what required to employ several particular steps from computer vision area.

As it is known, the smallest part of an image is a pixel. We cannot access information "between" pixel in usual. But there is a possibility to use some mathematical techniques to interpolate or approximate the brightness intensity among pixels and increase the detected points accuracy [3] [4].

Nowadays the camera is becoming a valuable sensor suitable for various kinds of measurements. The requirement of distance measuring or $3 \mathrm{D}$ position reconstructing is very often considered in computer or machine vision area and therefore widely used in many practical applications. In case of camera displacement estimation, the whole procedure is quite comprehensive and theory behind camera stereo pair configuration has to be applied. Therefore the theory of epipolar geometry and its relation to obtaining the 3D coordinates will be slightly mentioned to cover the theory behind the experiment described later.

\section{CORNER DETECTION}

There is no exact definition what the corner point is, but except many other statements it could be: point where at least two edges are intersected, point having the smallest radius of curvature or point around which is high change of brightness intensity in all directions.

Many corners detectors were invented over the years and the Harris corner detector [5] is one of the most common. The basic idea is to find the minimum of intensity difference between the chosen part of an image (marker as $W$ ) and the shift part of image $W$ in all directions. The first step is determination of matrix $A$ as it is shown in (1):

$$
A(x, y)=\sum_{W}\left[\begin{array}{l}
I_{x} \\
I_{y}
\end{array}\right]\left[\begin{array}{ll}
I_{x} & I_{y}
\end{array}\right]
$$

The variables inside are approximations of derivations (also referred as differences) in horizontal and vertical directions. The next step is determination of the cornerness matrix $C$. There are various formulas for calculation of matrix $C$ cornerness values published. The last step is looking for elements in matrix $C$ having the highest values (global and local thresholding). These points are stated as corners.

As it is obvious, this algorithm can be used to find corner points in pixel accuracy. Here it will be shortly mentioned two ways how to obtain the subpixel coordinates of corner points. These two approaches were also implemented in our comparison. For some other algorithms of subpixel corner detection see [6].

The first compared algorithm [7] was designed only for $x$ corner points detection, working directly with image brightness intensities and is based on the fact, that vector from the corner (marked $q$ ) to its adjacent area (marked $p$ ) should be perpendicular to the gradient of point $p$ as it is shown in the following formula:

$$
I\left(p_{i}\right)^{T} \cdot\left(q-p_{i}\right)=0
$$

The position of point $q$ is then solved through the iterations. 
The second method [8] primarily used for any kind of corners is refining the position of initially found corner point by fitting the quadratic curve to the corner strength function (cornerness map) in $x$ and $y$ direction separately. The approximation function is following: formula:

$$
h(x)=a x^{2}+b x+c
$$

Maximum of this function corresponds to subpixel corner coordinate in particular direction.

\section{THE PRESENTED APPROACH}

The our proposed approach here employs the cornerness map differently. The cornerness points in a chosen area surrounding the initially found corner point are considered as a $3 \mathrm{D}$ profile object. The key is to find the coordinates of its centroid point what can tell us the location of the expected corner point. The situation is illustrated in Figure 1.

Also this subpixel corner point determination is not computationally expensive. It could be computed by stating the weighted average for the $x$ and $y$ coordinates (cornerness values represents the weights coefficients and for better performance should be normalized) while the $z$ dimension is not taken into account.

\section{THE DISPLACEMENT DETERMINATION}

The whole process of getting the distance between two cameras in SI units was also described in [9] and consists of several steps.

\section{A. Camera Calibration}

Camera calibration [10] is primarily looking for quantities that affect the imaging process, such as focal length, principal point, skew factor and lens distortion. All these data are used later in distortion removal and epipolar geometry theory to provide us the better results.

\section{B. Feature Detection and Matching}

Feature detection and matching are one of the most crucial and important components of many computer vision algorithms. Basically the meaning of this step is the searching for interested parts of an image (features) which in case of two images contain the same scene can be correspondingly matched using so called feature descriptors. Amongst the common procedures can be counted SIFT [11] or SURF [12] algorithm for example.

\section{Image Undistortion}

By the definition the term distortion [13] is in photography generally representing the deformation and bending physically straight lines what makes them appear curly in images. These effects are very often in form of radial and tangential distortion and are mostly caused by lens and manufacturing errors. Because the distortion can be described as mathematical function using the information coming from camera calibration, the reversed mathematical operation called image undistortion is usually applied. The found corresponding points (in our case the corners) are because of that reason undistorted and processed in this form for the rest of the procedure.
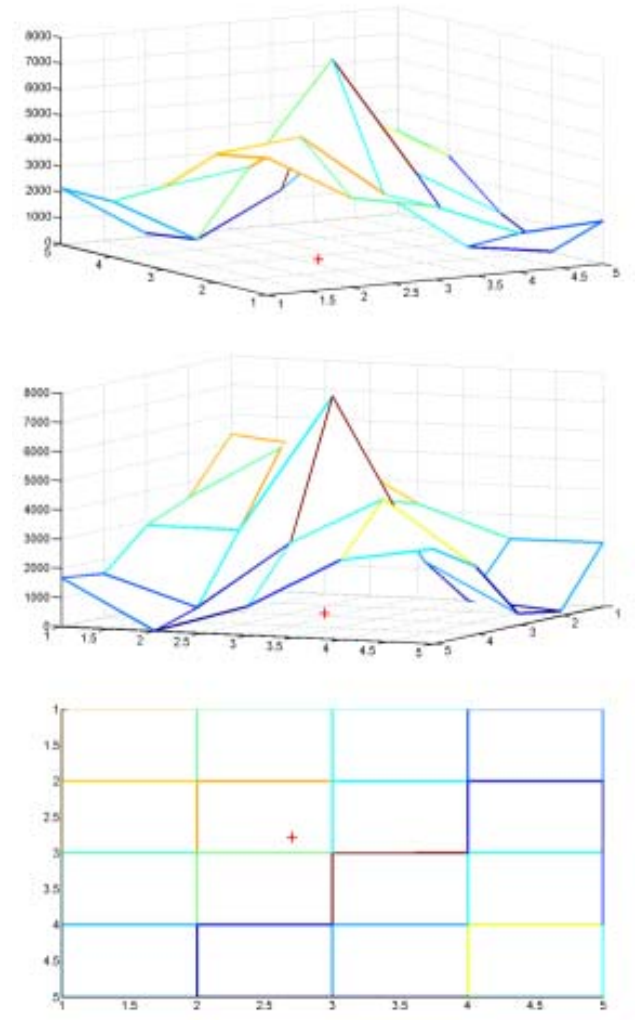

FIGURE I. CENTROID POSITION IN CORNERNESS PROFILE

\section{Essential Matrix}

In case of properly matched the corresponding points, the theory behind the epipolar geometry [14] is employed to extract the relative rotation and relative translation between both cameras (images) in stereo pair. As the first step the fundamental matrix has to be stated. This matrix describes the relation between the points in first image and so called epipolar lines in second image on which the set of corresponding points must lie. Using again the information from camera calibration and this matrix, the essential matrix can be computed.

\section{E. Decomposition of Essential Matrix}

There is possibility due its character to decompose the essential matrix into rotation matrix and translation vector what can fully describe the relative movement from one camera to another one in 3D Cartesian coordinate system. For that reason the SVD (singular value decomposition) technique [15] is usually used. But there is an issue. Due to fact that the objects having the different size and different scene distance can also look the same in image, the translation only relative up to scale can be obtained.

\section{F. Triangulation}

To obtain the points in 3D space, the triangulation using DLT algorithm [16] can be used. 
So the relation between image coordinates and 3D coordinates is shown in next formula:

$$
\left[\begin{array}{c}
u / w \\
v / w \\
w
\end{array}\right]=[K \llbracket R \mid t]\left[\begin{array}{c}
X \\
Y \\
Z \\
1
\end{array}\right]
$$

The symbols $u / w$ and $v / w$ represents 2D image pixel coordinates, $K$ is $3 \times 3$ intrinsic camera matrix (from camera calibration), $R \mid t$ is $3 \times 4$ extrinsic camera matrix (from essential matrix decomposition) and $X, Y, Z$ are $3 \mathrm{D}$ world coordinates of chosen point.

The reason for triangulation in describing displacement determination is that after the 3D points using $R$ matrix and $t$ vector are stated, the reprojection back into $2 \mathrm{D}$ image space using previous formula can gives us information about decomposition validity.

\section{G. Image Rectification}

Image rectification [17] is basically a transformation process used to project two or more images onto a common image plane. The found translation vector as the information about mutual cameras position is used in rectifying process. It is not necessary to rectify the whole image due to fact that the rectifying transformation only for detected corners is for our purposes sufficient enough. Because of rectification both image planes are parallel and the depth triangulation is therefore much easier. Another parameter needed for 3D depth or camera displacement calculation is pixel distance between corresponding points.

\section{H. Disparity}

If we assume calibrated and rectified stereo pair, disparity [18] by definition measures the displacement of a point between two images in pixels. This situation is illustrated in Figure 2. It is obvious that in case of close points in scene there is large displacement and small displacement for far points respectively. The depth $Z$ calculation using disparity $d$ information is computed like this:

$$
Z=\frac{f \cdot B}{d}
$$

The sign $f$ is stand for focal length in pixels we found during camera calibration process. The $B$ as the baseline also represents the displacement between both stereo pair cameras in SI units.

Due to the mentioned fact that objects having different size and scene distance can look similarly or even the same in image, we need to know some intrinsic prior information like focal length $f$ and depth $Z$ (this information may easily be obtained using another sensors for example) in rectified 3D coordinate system to be able successfully determine real displacement $B$ between cameras in SI units. In case this procedure is used for navigation purposes, the $Z$ prior information is needed only in first step, for further steps (applying described procedure repeatedly) is possible to calculate another depth coordinates by using data (rotation, translation and so on) gathered during the process.

\section{EXPERIMENTAL TESTS}

For the purpose to demonstrate the possibility of mutual camera distance estimation and the direct impact of accuracy of corner points position on it, the camera stereo pair configurations using calibrated camera were prepared.

All particular presented steps were followed to determine the position displacement of the same camera. The both camera positions, which were mutually translated and rotated, give us the stereo pair configuration.

The camera calibration process is illustratively shown in Figure 3. and the example of the camera stereo pair preparation is in Figure 4. The detected points correspondingly matched are illustrated in Figure 5.

For the easier detection of corner points the chessboard pattern was used. These points were used to determine the fundamental matrix and during the next steps both sets of points were rectified. There rectified points were further investigated to find the displacement through disparity computation. Since the prior information is necessary, the real scene depth $Z$ value in $\mathrm{cm}$ unit considers the rectified coordinate system was known for all these points.

The positions of corner points were found using all mentioned approaches: Harris detector (marked as $P$ ), subpixel approach based on orthogonal vectors $(S A)$, subpixel algorithm based on curve overlapping $(S B)$ and our presented method $(S C)$.

To make the analysis more robust, three image resolutions were processed: $2560 \times 1920,1280 \times 960$ and $640 \times 480$ pixel size. The multiple image pairs were tested with mutual camera distance $30 \mathrm{~cm}$. Also the many corner points combinations (fundamental matrix computation and so on) for every image pair were used.

\section{EXPERIMENTAL RESULTS}

The results related to describing test were about stability investigation. As this whole displacement estimation process is a set of theoretic mathematical algorithms and approaches, it could easily give us physically not possible results. That's why we decided to use our own filter to recognize if the results are acceptable or not.

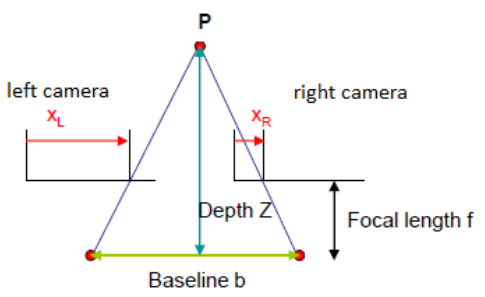

FIGURE II. THE SITUATION IN RECTIFIED STEREO PAIR 


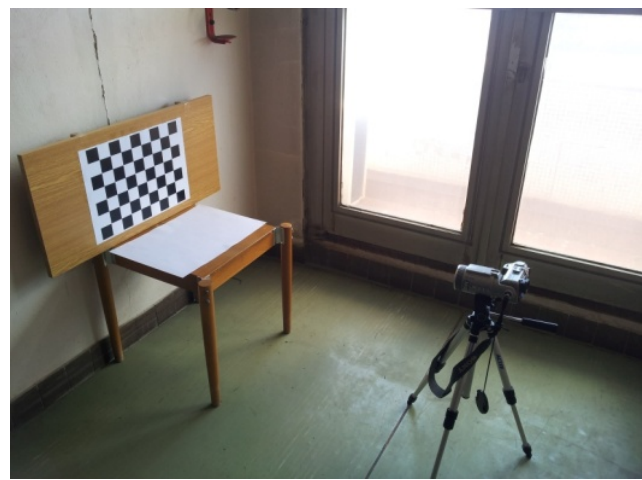

FIGURE III. THE CAMERA CALIBRATION

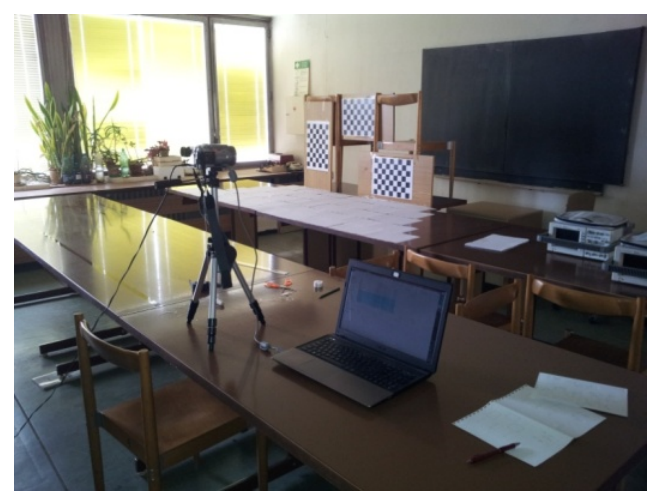

FIGURE IV. THE STEREO PAIR CONFIGURATION

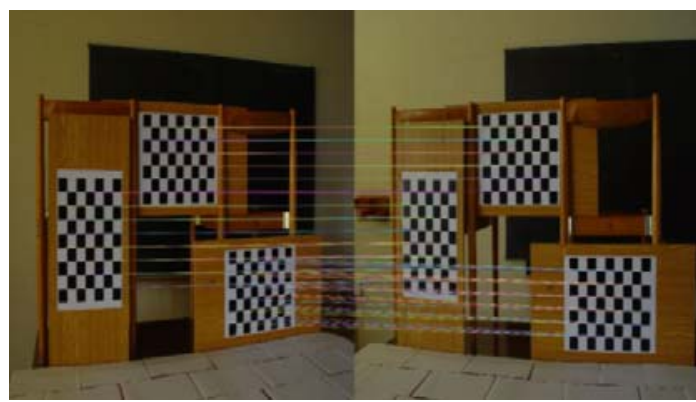

FIGURE V. THE MATCHED CORNER POINTS IN IMAGE STEREO PAIR

The first criterion was the fact, that all the corresponding points (usually 144) triangulated using the found matrix $R$ and the vector $t$ had to have the positive depth $Z$ coordinate. If they hadn't, the tested stereo pair was denied. Another criterion was the difference between the found and the real camera displacement. Because the real camera shift as the ground truth was known and, in case that the averaged computed shift from all points involved was off more than $50 \%$ in the contrary to real one, again the tested stereo pair was denied.

These stability results are listed in Table 1.

There are 3 types of result values in table. The first one marked as ratio represents the ratio between valid and all tested configurations. All resulted accepted displacement were averaged and also the standard deviations were computed (mean and std).
TABLE I. THE EXPERIMENT RESULTS

\begin{tabular}{|c|c|c|c|c|}
\hline \multirow{2}{*}{ Method } & \multirow{2}{*}{ Stats } & \multicolumn{3}{|c|}{ Resolution [pixel] } \\
\hline & & $640 \times 480$ & $1280 \times 960$ & $2560 \times 1920$ \\
\hline \multirow{3}{*}{$\mathbf{P}$} & Mean [mm] & 28.1969 & 28.4884 & 30.2809 \\
\hline & Std [mm] & 6.67105 & 5.55875 & 5.58945 \\
\hline & Ratio & 0.181909 & 0.322455 & 0.373909 \\
\hline \multirow{3}{*}{ SA } & Mean [mm] & 34.1602 & 34.8483 & 33.7326 \\
\hline & Std [mm] & 4.07529 & 3.93505 & 3.97507 \\
\hline & Ratio & 0.411364 & 0.455727 & 0.460273 \\
\hline \multirow{3}{*}{ SB } & Mean [mm] & 33.007 & 33.9673 & 33.3181 \\
\hline & Std [mm] & 5.44053 & 4.63532 & 4.84324 \\
\hline & Ratio & 0.379182 & 0.429182 & 0.420273 \\
\hline \multirow{3}{*}{ SC } & Mean [mm] & 30.201 & 29.438 & 31.186 \\
\hline & Std $[\mathrm{mm}]$ & 6.16958 & 5.39716 & 5.41697 \\
\hline & Ratio & 0.373455 & 0.392636 & 0.394455 \\
\hline
\end{tabular}

As you can see, the subpixel approaches provides us better stability results as original pixel detector. Also the our proposed method gives the similar results as another subpixel ones.

What is interested is the fact, that in table it is possible to see, that in case of low resolution images the benefit of employing subpixel detection is higher than in case of high resolution ones.

\section{CONCLUSION}

This paper has dealt with comparison between the presented subpixel corner detection approach based on searching for centroid position of 3D profile function represents the cornerness map around the initial corner point and another pixel and subpixel methods. As the subject of comparison the determination procedure of the mutual displacement between two corresponding cameras using image stereo pair was chosen.

The first parts contain the theory behind pixel and subpixel detection and particular detection methods.

The next part deals with explanation of our proposed subpixel approach.

Then the whole procedure of camera shift estimation was discussed and all particular algorithm steps were briefly explained.

The experimental test which was performed according the described procedure was presented in another section. All procedure steps together with pixel and subpixel detections were followed to determine the shift between two cameras. Also the intrinsic information such as scene depth coordinates for investigated points were known.

The analysis was about the stability evaluation. Using the restricted filter to accept or dismiss the results, we compared the differences between particular corner detectors. It was found, that subpixel detector provides us better results than usual pixel one. Also the our presented approach gives the comparable results to the other subpixel methods. It was also possible to see, that the benefit of subpixel detection is more significant in case of lower resolution images than in higher ones. 
The results so far indicate the possible convenience of usage our proposed subpixel method in area of corner detection, but the deeper analysis has to be performed to get the final answer.

\section{ACKNOWLEDGMENT}

This work was supported by the Slovak Research and Development Agency under the contract No. APVV-0469-12.

\section{REFERENCES}

[1] L. Šroba, R. Ravas and J. Grman, A New Approach to Obtain Subpixel Corner Point Coordinates Based on Position from Cornerness Map Refinement, Journal of Multidisciplinary Engineering Science and Technology (JMEST), Vol. 3 Issue 4, 2016, 4641-4643.

[2] L. Šroba, R. Ravas and J. Grman, A New Approach to Obtain Corner Point Coordinates in Subpixel Accuracy, In CMSAM 2016: International conference on computational modeling, simulation and applied mathematics, Bangkok Thailand, 2016, 204-208.

[3] Y. Qiao, Y. Tang and J. Li, "Improved Harris sub-pixel corner detection algorithm for chessboard image”, Conference on Measurement, Information and Control (ICMIC) Volume 02, 2013, pp. 1408-1411.

[4] N. Chen, J. Wang, L. Yu and Ch. Su, "Sub-pixel Edge Detection of LED Probes Based on Canny Edge Detection and Iterative Curve Fitting”, International Symposium on Computer, Consumer and Control (IS3C), 2014, pp. 131-134.

[5] Ch. Harris and M. Stephens, "A Combined Corner and Edge Detectors”, In Alvey Vision Conference, 1988, pp. 147-152.

[6] L. Sroba and R. Ravas, "The subpixel X-corners detectors and their window shift robustness comparison”, ELITECH '12, 14th Conference of Doctoral Students, ISBN 978-80-227-3705-0, 2012.

[7] Z. Weixing, M. Changhua, X. Libing, L. Xincheng, A fast and accurate algorithm for chessboard corner detection, CISP $2^{\text {nd }}$ International Congress on, Image and Signal Processing (2009) 1-5.

[8] M. Rea, D. McRobbea, D. Elhawary, Z. Tse, M. Lamperth, I. Young, Sub-pixel localization of passive micro-coil fiducial markers in interventional MRI, MAGMA (2009), 2009.

[9] L. Sroba, R. Ravas, Determination of displacement between two cameras using corresponding image stereo pair, $16^{\text {th }}$ Conference of Doctoral Students ELITECH (2014).

[10] D. C. Brown, Close-Range Camera Calibration, Photogrammetric Eng. Vol. 37 (1971) 855-866.

[11] D. G. Lowe, Distinctive Image Features from Scale-Invariant Keypoints, International Journal of Computer Vision Volume 60 Issue 2 (2004) 91110

[12] H. Bay, A. Ess, T. Tuytelaars, L. Van Gool, Speeded-Up Robust Features (SURF), Computer Vision and Image Understanding Volume 110 Issue 3 (2008) 346-359.

[13] D. C. Brown, Decentering distortion of lenses, Photogrammetric Eng Vol. 32 (1966) 444-462.

[14] R. Hartley, A. Zisserman, Multiple View Geometry in Computer Vision, Cambridge University Press (2004) 239-259.

[15] W. Wang, H. Tsui, A SVD decomposition of essential matrix with eight solutions for the relative positions of two perspective cameras, $15^{\text {th }}$ International Conference on Pattern Recognition (2000) 362-365.

[16] R. Hartley, A. Zisserman, Multiple view geometry in computer vision, 2ndedition, Cambridge University Press, 2003, 89-90.

[17] D. Oram, Rectification for any epipolar geometry, British Machine Vision Conference (2001) 653-662.

[18] N. Navab, Ch. Unger, Rectification and disparity, Computer Aided Medical Procedures in Technical University Munich. 